\title{
Venlafaxine overdose treated with extracorporeal life support
}

\author{
Laurel Murphy MD, Jack Rasmussen MD, Nancy G. Murphy MD CM
}

- Cite as: CMAJ 2021 February 1;193:E167-70. doi: 10.1503/cmaj.201318

A n 18-year-old woman was found at home by her mother after an intentional polydrug overdose. The time of ingestion was estimated to be between 2 and 5 hours before the patient's mother discovered her. The patient had received a diagnosis of borderline personality disorder and had a history of overdose attempts that did not require admission to hospital. Through cross-referencing of her pill bottles and known prescriptions, we calculated that the ingestion included the following: venlafaxine XR $150 \mathrm{mg}$ $\times 120$ tablets $(18000 \mathrm{mg})$, fluoxetine $10 \mathrm{mg} \times 30$ tablets $(300 \mathrm{mg}$ ) and diazepam $5 \mathrm{mg} \times 10$ tablets $(50 \mathrm{mg})$.

While transporting the patient to the hospital, paramedics noted seizure-like activity that aborted without treatment. In the emergency department, the patient was drowsy and intermittently following commands. Her temperature was $34.9^{\circ} \mathrm{C}$, heart rate 109 beats/min, respiratory rate 24 breaths/min, blood pressure $108 / 72 \mathrm{~mm} \mathrm{Hg}$, and her oxygen saturation was $96 \%$ on room air. Her pupils were dilated. There was no clonus, rigidity or hyperreflexia. Blood glucose was normal, and a blood toxicology screen was negative for acetylsalicylic acid, acetaminophen and alcohol. We did not perform a urine drug screen.

The initial electrocardiogram (ECG) is shown in Figure 1. The patient had a seizure shortly after arrival and was treated with lorazepam $1 \mathrm{mg}$, administered intravenously. We contacted our provincial poison centre for advice on patient management and monitoring; decontamination was contraindicated, owing to her compromised, unprotected airway. Over the next 6 hours, the patient had an additional 3 seizures and her temperature rose to $38.5^{\circ} \mathrm{C}$. Given her escalating oxygen requirements, we decided to intubate her, after which she received infusions of fentanyl, midazolam and norepinephrine.

Three hours after intubation, the patient became more tachycardic, as the ECG in Figure 2 shows. She subsequently developed a wide complex rhythm (Figure 3), and her blood pressure decreased to $66 \mathrm{~mm} \mathrm{Hg}$ by palpation. Cardioversion was unsuccessful. Shortly after this, the patient had a 9-minute pulseless electrical activity arrest, followed by another of 7 minutes. A bedside ultrasound by the on-call intensivist showed profound biventricular cardiac dysfunction with an estimated left ventricular ejection fraction of less than $10 \%$, despite high-dose norepinephrine and epinephrine at the time of the ultrasound.

\section{KEY POINTS}

- Venlafaxine overdoses can be life threatening.

- Signs of toxicity include seizure and prolongation of the QRS and QTC.

- Large venlafaxine ingestions can cause a severe, reversible cardiomyopathy requiring extracorporeal life support.

- Severe poisonings should prompt a discussion with the provincial poison centre regarding gastrointestinal decontamination and management.

The patient was transferred to the intensive care unit, where she required high-dose vasopressors and inotropes and had an immeasurably high lactate. She developed progressive hypotension, and a transesophageal echocardiogram showed a calculated ejection fraction of $6 \%$. She then received extracorporeal life support (ECLS) as a bridging strategy, which was combined with continuous renal replacement therapy for acute kidney injury. She had biochemical evidence of acute hepatic injury with hypoglycemia requiring a glucose infusion.

Over the next week, the patient's cardiac function gradually recovered. Her echocardiogram on day 4 after admission to hospital showed improvement in the ejection fraction to $25 \%-30 \%$; the left ventricular systolic function was still severely reduced. By postadmission day 7 , her cardiac function had recovered to normal, with a normal ejection fraction. On day 8 , she was successfully weaned from ECLS and decannulated. Until this point in her admission, she had received no enteral feeds or medications, and had not had a bowel movement. Gastric aspirates at this time showed numerous bead-like pill fragments (Figure 4). In consultation with the poison centre, delayed gastric lavage was recommended, owing to lack of availability of immediate endoscopy; whole-bowel irrigation could not be performed because of a lack of peristalsis. Many beads were aspirated. Upper endoscopy performed the following day was normal, apart from a few residual beads.

She was extubated on day 13 after admission. Her renal function recovered, and she was transitioned off hemodialysis. Neurologically, she achieved full recovery to her preadmission baseline. She was followed by the psychiatry team as an inpatient and was connected with dialectical behaviour therapy as an outpatient. She was discharged from hospital 43 days after admission. 


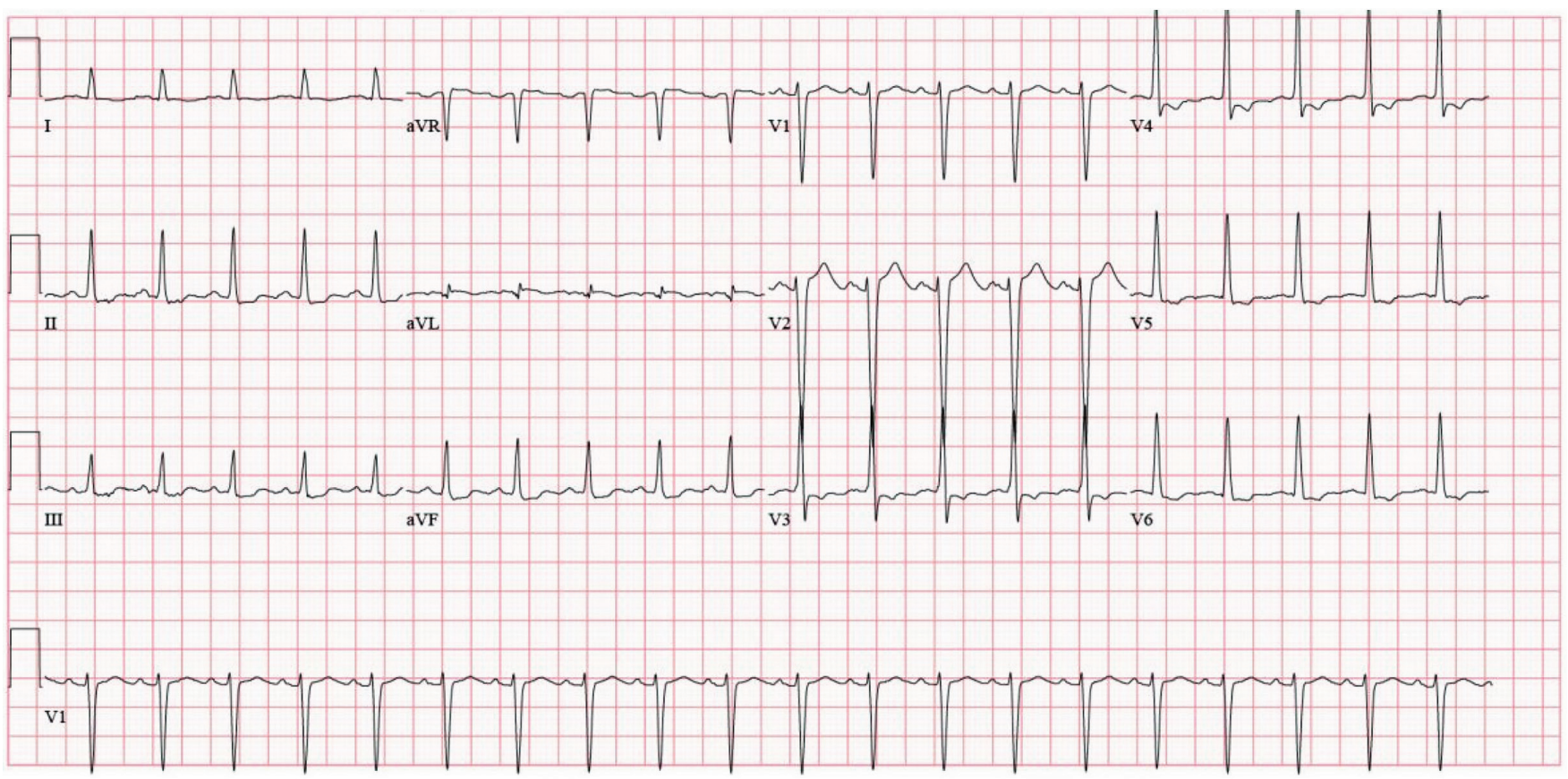

Figure 1: Electrocardiogram of an 18-year-old woman on presentation to the emergency department: QTc interval 427 ms, QRS interval 100 ms.

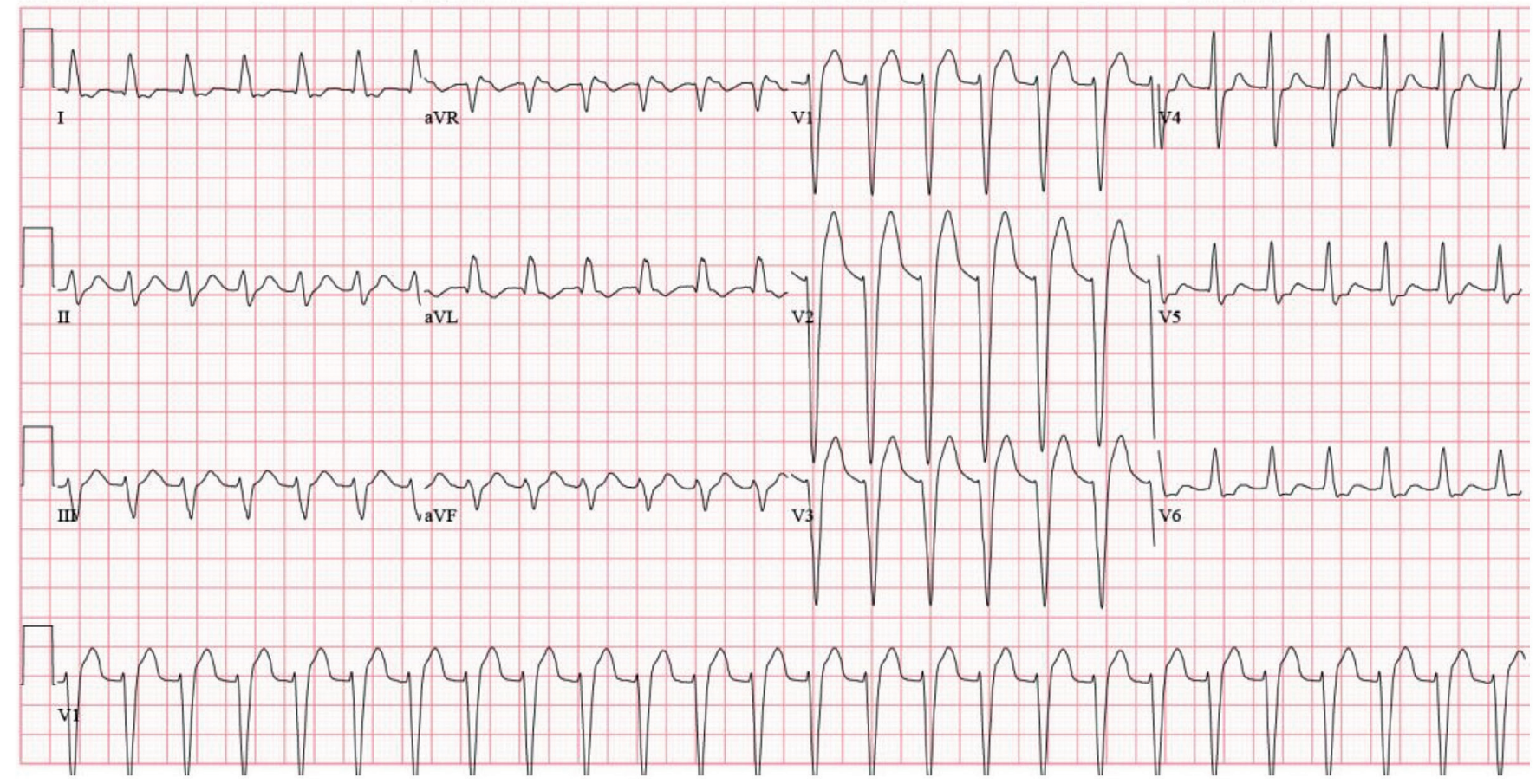

Figure 2: Electrocardiogram about 10 hours after the patient's arrival at the emergency department: QTc interval 531 ms, QRS interval 160 ms.

\section{Discussion}

Venlafaxine is a serotonin-norepinephrine reuptake inhibitor and is commonly prescribed for moderate to severe depression. It is available in immediate- or extended-release formulations. The extended-release formulation is supplied as either a pressed capsule, or a gelatin capsule filled with white to off-white beads. Venlafaxine XR is the only ingestant in this case that is formulated containing beads. Although the likelihood is high that the beads retrieved from the gastrointestinal tract were venlafaxine $\mathrm{XR}$, they were not tested for identification in this case. The toxicity of venlafaxine is less than that of tricyclic antidepressants, but significantly higher than the selective serotonin reuptake inhibitors (SSRIs). ${ }^{1}$ Among non-SSRI antidepressants, venlafaxine has one of the highest risks of QT prolongation in overdose, with the associated risk of sudden cardiac death caused by torsades de pointes. ${ }^{2}$ 


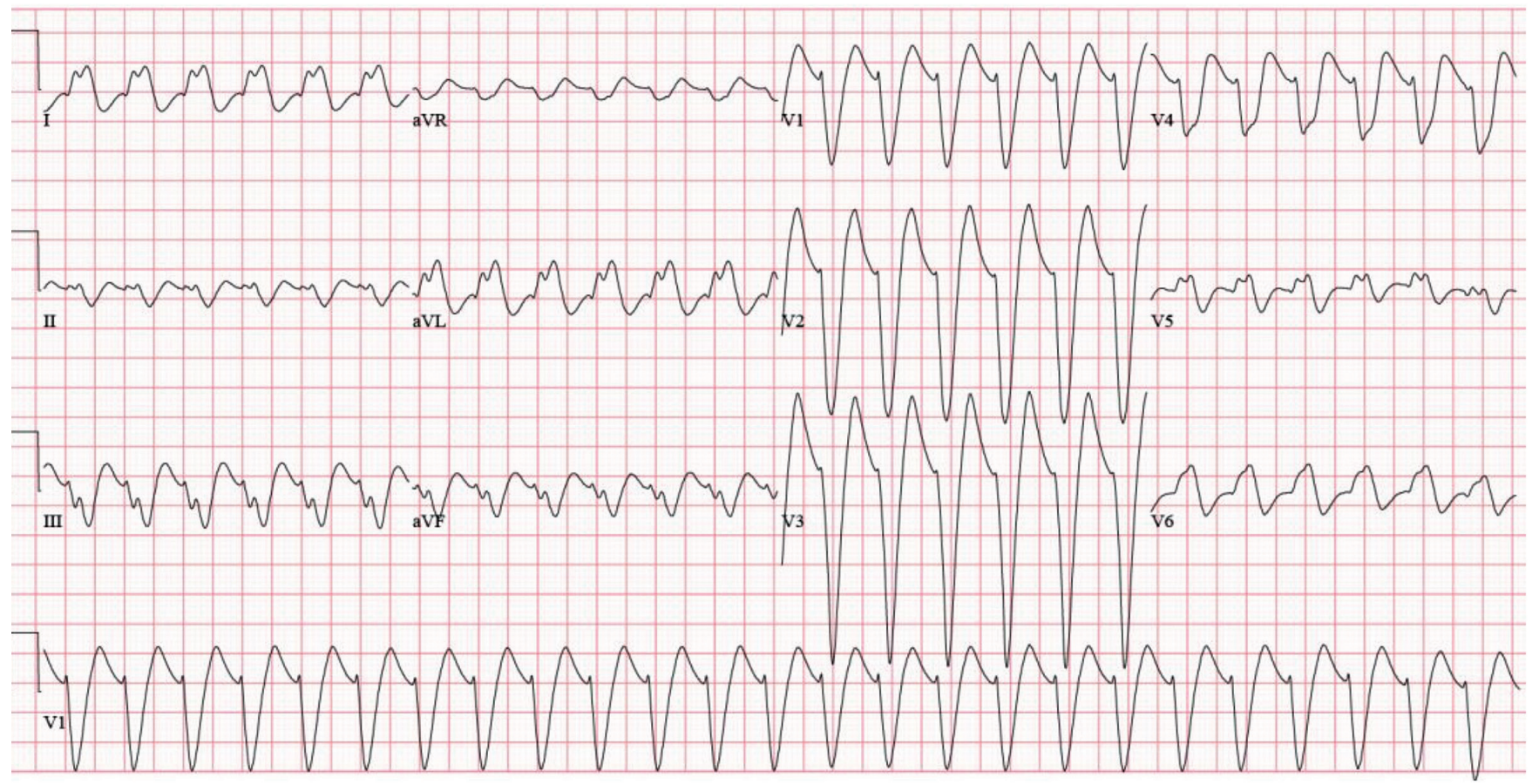

Figure 3: Wide complex tachycardia in the same patient.

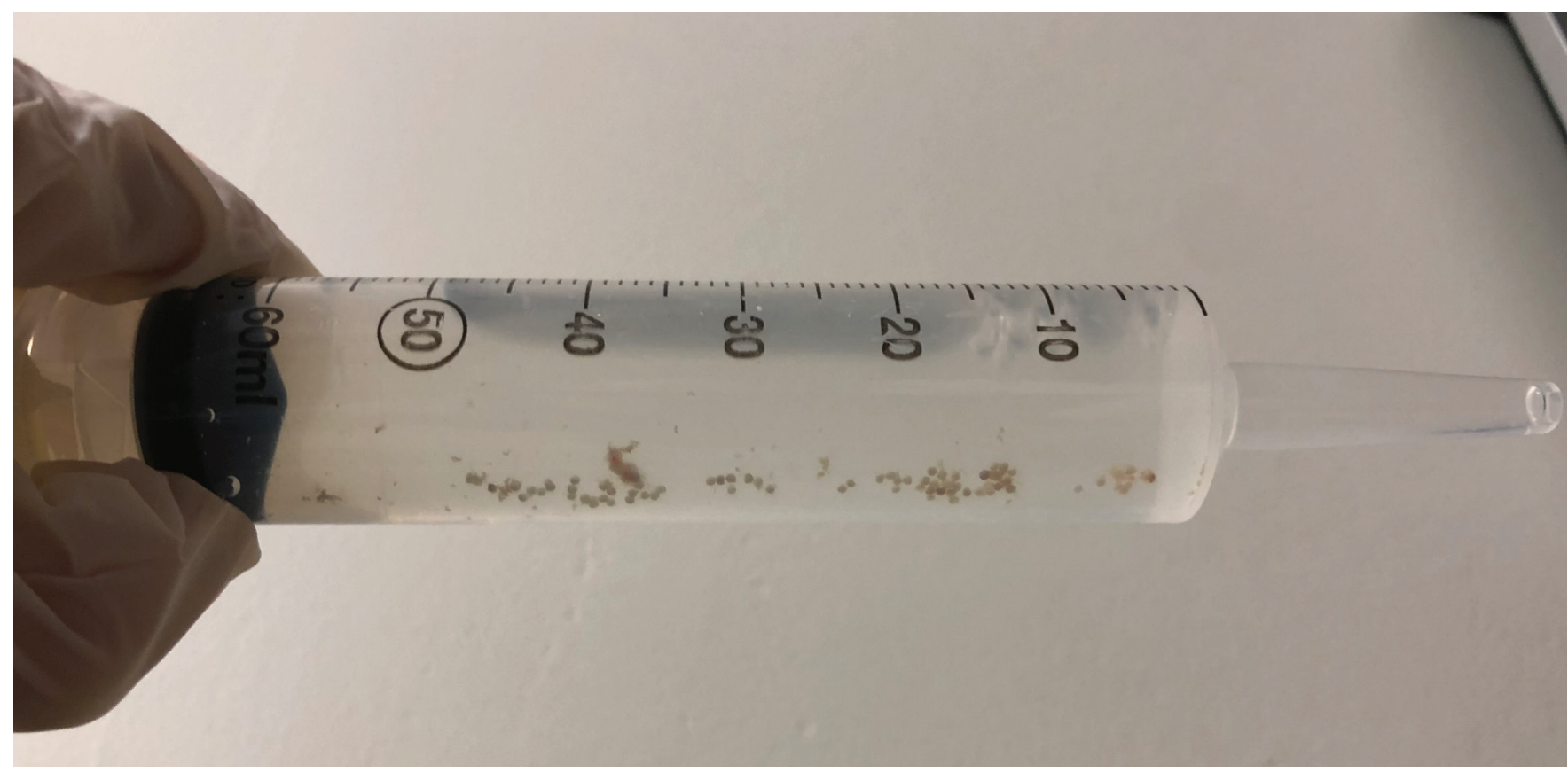

Figure 4: Contents of gastric lavage performed on day 8 .

\section{Seizure}

Seizure is a common sequela of venlafaxine overdose, and there have been uncommon reports of venlafaxine-associated seizures at therapeutic doses. The California Poison Control System's retrospective review of 953 venlafaxine ingestions found that seizures occurred in $12.9 \%$ of cases. ${ }^{3}$ Current poison centre recommendations, although variable, suggest a 6-hour observation for immediate-release formulations and 18- to 24-hour observation for ingestions of sustained-release formulations. ${ }^{3}$

\section{Cardiac toxicity}

Venlafaxine overdose is associated with tachycardia, dosedependent QT prolongation and QRS prolongation, all of which were demonstrated in this patient. Massive ingestions of more than $8 \mathrm{~g}$ may be particularly susceptible to cardiac toxicity ${ }^{4}$ (our patient ingested $18 \mathrm{~g}$ ). Our case shows a clear progression of the QRS and QTc intervals. On presentation, both intervals were normal, with a QTc of $427 \mathrm{~ms}$ and a QRS of $100 \mathrm{~ms}$ (Figure 1). The ECG performed 10 hours after the patient's arrival in the emergency 
department shows an increase in the QTc to $531 \mathrm{~ms}$ and widening of the QRS to $160 \mathrm{~ms}$ (Figure 2).

Venlafaxine overdose has been associated with numerous reports of reversible cardiomyopathy. Our patient showed an extreme example of this, requiring 8 days of ECLS support while her cardiac function recovered. To our knowledge, 3 other reports in the literature have reported successfully using ECLS for similarly massive venlafaxine ingestions ( $17.5 \mathrm{~g}$ to $20 \mathrm{~g}$ ). ${ }^{5}$ The mechanism of cardiac toxicity is not entirely understood, but 2 theories have been suggested. The first is that venlafaxine inhibits sodium channels in ventricular myocytes in a concentration-dependent manner, resulting in myocardial stunning. ${ }^{6}$ The characteristics of this sodium channel blockade are different from that observed with tricyclic antidepressants or class I antiarrhythmics. The second proposed mechanism of cardiac toxicity is that of excess adrenergic stimulation, resulting in myocardial stunning similar to Takotsubo cardiomyopathy. ${ }^{7}$

\section{Gastrointestinal decontamination}

Overdose of an oral drug with extended-release properties may result in a substantial quantity of unabsorbed drug remaining in the gastrointestinal tract for a long time, and although gastrointestinal decontamination is controversial, there are scenarios where it is beneficial. ${ }^{8}$ The overarching principle is to reduce or prevent absorption of drug, thereby reducing potential toxic effects. In light of limited evidence for efficacy, the risks of performing decontamination must be weighed against the potential benefits in all cases. Generally, contraindications for all forms of gastrointestinal decontamination include an unprotected airway, repeated vomiting, a nonintact gastrointestinal tract (e.g., ileus, obstruction), hemodynamic instability and a high risk of aspiration (e.g., hydrocarbon ingestion). ${ }^{9}$

Gastric lavage is a rarely used method of gastrointestinal decontamination that has largely been supplanted by less invasive methods, such as activated charcoal and whole-bowel irrigation. In recent years, endoscopic lavage has been described to remove retained gastric pill material, but no clear recommendations exist to guide selection of patients for this procedure. In our patient, although a large amount of residual pill material was retrieved 8 days after ingestion, it is unclear whether there was any active drug remaining. However, the very presence of this pill residue illustrates that in some cases of large ingestions of modified-release drugs, there can be delayed and prolonged absorption; addressing the possibility of decontamination early on in the clinical course should be undertaken in consultation with the poison centre. Unfortunately, our patient had contraindications to decontamination on initial presentation (unprotected airway) and after intubation (hemodynamic instability), which highlights the limitations of this intervention.

\section{Conclusion}

Venlafaxine overdose can result in life-threatening toxicity, most often in the form of cardiac collapse. In large ingestions, patients may develop a severe but reversible cardiomyopathy that requires
ECLS support. In light of this, we would suggest that any patient who ingests massive quantities of venlafaxine $(>8-10 \mathrm{~g})$ should have some form of echocardiographic assessment early in their presentation, and that it be repeated if the patient's hemodynamics begin to worsen. Given the life-saving role of ECLS in this patient, we also suggest that in massive overdoses, patients should be transferred to a centre where ECLS can be provided if needed. Early consultation with a poison centre is strongly recommended to discuss options for gastrointestinal decontamination and for expertise in anticipating and managing severe drug toxicity.

\section{References}

1. Hawton K, Bergen H, Simkin S, et al. Toxicity of antidepressants: rates of suicide relative to prescribing and non-fatal overdose. $\mathrm{Br} J$ Psychiatry 2010;196:354-8.

2. Jasiak NM, Bostwick JR. Risk of QT/QTc prolongation among newer non-SSRI antidepressants. Ann Pharmacother 2014;48:1620-8.

3. Vo KT, Merriman AJ, Wang RC. Seizure in venlafaxine overdose: a 10-year retrospective review of the California poison control system. Clin Toxicol (Phila) 2020;58:984-90.

4. Isbister GK. Electrocardiogram changes and arrhythmias in venlafaxine overdose. Br J Clin Pharmacol 2009;67:572-6.

5. Marquetand C, Langer HF, Klein JP, et al. The use of extracorporeal life support in a patient suffering from venlafaxine intoxication: a case report. J Crit Care Med (Targu Mures) 2020;6:120-3.

6. Khalifa M, Daleau P, Turgeon AJ. Mechanism of sodium channel block by venlafaxine in guinea pig ventricular myocytes. J Pharmacol Exp Ther 1999;291: 280-4.

7. Vasudev R, Rampal U, Patel H, et al. Selective serotonin-norepinephrine reuptake inhibitors-induced takotsubo cardiomyopathy. N Am J Med Sci 2016;8:312-5.

8. Livshits Z, Sampson BA, Howland MA, et al. Retained drugs in the gastrointestinal tracts of deceased victims of oral drug overdose. Clin Toxicol (Phila) 2015;53: 113-8.

9. Benson BE, Hoppu K, Troutman WG, et al. Position paper update: gastric lavage for gastrointestinal decontamination. Clin Toxicol (Phila) 2013;51:140-6.

\section{Competing interests: None declared.}

This article has been peer reviewed.

The authors have obtained patient consent.

Affiliations: Departments of Emergency Medicine and Critical Care (L. Murphy), and Surgery; Division of Plastic Surgery, Department of Critical Care (Rasmussen); Department of Emergency Medicine and IWK Regional Poison Centre (N. Murphy), Dalhousie University, Halifax, NS

Contributors: All of the authors contributed to the conception and design of the work, drafted the manuscript, revised it critically for important intellectual content, gave final approval of the version to be published and agreed to be accountable for all aspects of the work.

Content licence: This is an Open Access article distributed in accordance with the terms of the Creative Commons Attribution (CC BYNC-ND 4.0) licence, which permits use, distribution and reproduction in any medium, provided that the original publication is properly cited, the use is noncommercial (i.e., research or educational use), and no modifications or adaptations are made. See: https://creativecommons.org/licenses/by-nc-nd/4.0/

Correspondence to: Laurel Murphy, laurel.murphy@dal.ca 